\title{
(Inter-)Kulturelle Kohäsion in grenzübergreifenden Kultur- und Sprachräumen
}

Online publiziert: 9. Dezember 2011

(C) Springer-Verlag 2011

„Kohäsion“, deutsch „Zusammenhalt“, ist ein Begriff, der sich über die Natur-, Sozial- und Sprachwissenschaften hinaus längst auch das Feld der Politik erobert hat. Angesichts der in jüngerer Zeit deutlich zunehmenden Zentrifugalkräfte, die nicht nur auf dem europäischen Wirtschafts- und Finanzsektor erkennbar sind, hat die Frage der Kohäsion gerade in der Europäischen Union weiter an Bedeutung gewonnen. Dies zeigt sich auch daran, dass seit dem Vertrag von Lissabon von 2007 nicht nur die wirtschaftliche und soziale, sondern auch die territoriale Kohäsion zu den Zielen der Union zählt (Art 3 EUV).

Ein Instrument, das dazu beitragen soll, vor allem die zwischen den einzelnen EU-Mitgliedstaaten bestehenden wirtschaftlichen und sozialen Disparitäten längerfristig auszugleichen, um dadurch gerade über die „Nahtstellen“ der Staatsgrenzen hinweg den EU-internen Zusammenhalt zu stärken, ist der 2006 via Verordnung geschaffene Europäische Verbund für territoriale Zusammenarbeit EVTZ. So besteht durch die Rechtsform des EVTZ erstmals die Möglichkeit der formalisierten und institutionalisierten grenzüberschreitenden Zusammenarbeit zwischen Gebietskörperschaften.

Walter Obwexer und Esther Happacher haben in EJM 2-1010 die genaueren rechtlichen Rahmenbedingungen und Voraussetzungen für die Gründung eines EVTZ erörtert und diese auf den konkreten Fall des in statu nascendi befindlichen (und inzwischen weit im Gründungsprozess fortgeschrittenen) EVTZ „Euregio Südtirol/ Alto Adige, Tirol und Trentino“ angewendet. Sie kommen dabei zu dem Schluss, dass der EVTZ sich zwar nicht, ,als spezifisches Instrument des Minderheitenschutzes eignet“, da dessen mögliche „Aufgaben auf den wirtschaftlich-sozialen Zusammenhalt begrenzt sind.“ Zugleich stellen sie aber fest, dass ein EVTZ, in seinem territorialen Geltungsbereich den Schutz von Volksgruppen bzw Minderheiten ergänzen und unterstützen" kann.

Alice Engl und Andreas Eisendle knüpfen in ihrem Beitrag direkt an diese Feststellungen an, indem sie zunächst auf die zwar nicht minderheitenspezifischen, aber doch auch minderheitenrelevanten Rahmenbedingungen des EVTZ hinweisen und 
dann die mögliche Minderheitenrelevanz eines EVTZ anhand der Praxis zweier 2008 gegründeter Verbunde, jenem des EVTZ Eurométropole Lille-Kortrijk-Tournai im französisch-belgischen Grenzraum und jenem des EVTZ Ister-Granum entlang der slowakisch-ungarischen Grenze eindrucksvoll illustrieren.

Die beiden Fallbeispiele legen zugleich offen, dass grenzübergreifende Sprachund Kulturräume durch ihre Bewohner über ein Brückenpotential verfügen, das im Rahmen der grenzüberschreitenden Zusammenarbeit einerseits zur Stärkung der betreffenden Sprachgruppen und Minderheiten - durch Ankoppelung an ihr kulturell-sprachlich verwandtes „Mutterland“ (oder kin state) - und zugleich zur Stärkung des interkulturellen Dialogs im Sinne einer umfassenden, nicht nur wirtschaftlichen und sozialen, sondern im „Nebenprodukt“ auch (inter-)kulturellen Kohäsion genutzt werden kann. Mit anderen Worten: Auch hier zeigt sich ein Mehrwertpotential von Minderheiten, von welchem alle Beteiligten profitieren können. ${ }^{1}$

Ein weiterer wichtiger, sich auch aus den beiden Fallbeispielen ergebender Aspekt ist dabei allerdings nicht zu vergessen: Es gilt, solche Potentiale, wie sie sich hier zB aus dem Rechtsinstrument des EVTZ in Verbindung mit dem Vorhandensein eines grenzübergreifenden Sprach- und/oder Kulturraumes ergeben, tatsächlich zu nutzen. Dies unterstreichen auch Engl und Eisendle, indem sie darauf verweisen, dass in beiden Fallbeispielen ,die Partner bereits vor der Verabschiedung der EVTZ-Verordnung ihr Interesse und den Willen [Hervorhebung d Verf] zur Zusammenarbeit zeigten“. Die genannten „Partner“ umfassen aber die politische ebenso wie die gesellschaftliche Ebene, konkret also die staatlich-nationalen, regionalen und/oder lokalen Akteure sowie die entsprechenden Mehrheits- und Minderheitsbevölkerungen.

Eine solche Eigenleistung, die Entwicklung eines (kollektiven) Willens zur Nutzung vorhandener rechtlicher und faktischer Möglichkeiten sowie die daraus folgende Bereitschaft, die eigenen (gruppen-)spezifischen Interessen im demokratisch-rechtsstaatlichen Rahmen zu vertreten und umzusetzen, kann insbesondere für kleine und womöglich noch in Streulage siedelnde Minderheiten zur kulturellen Überlebensfrage werden - ist es doch gerade die kulturell-sprachliche Eigenheit, das „Anderssein“ der betreffenden Gruppen und ihrer Angehörigen in Verbindung mit ihrer Kleinheit in Relation zur Mehrheitsbevölkerung, das diese schon ex definitione zu Minderheiten werden lässt. Kommt zu dieser relativen Kleinheit noch eine absolute in dem Sinne hinzu, dass eine Minderheit weniger als 300.000 oder gar weniger als 50.000 Angehörige zählt, so ist nach Meinung von Fachleuten die Überlebensfähigkeit der betreffenden Minderheitensprachen akut in Frage gestellt. ${ }^{2}$

\footnotetext{
${ }^{1}$ Ausführlich dazu unter dem Blickwinkel „,wirtschaftlicher Mehrwert durch Minderheiten“ EJM 22010, in welchem - ua aus Anlass entsprechender Europarat-Dokumente aus den Jahren 2009 bzw 2010 - die Zusammenhänge zwischen der Förderung von Minderheitensprachen, regionaler Selbstverwaltung, grenzüberschreitender Zusammenarbeit und wirtschaftlichem Mehrwert erörtert sind (vgl die betreffenden Einzelbeiträge sowie das Vorwort). Grundlegend dazu auch Matthias Theodor Vogt: Mehrwert durch Minderheiten? Das VIII. Collegium Pontes Görlitz- Pontes Görlitz-Zgorzelec-Zhořelec, in EJM 2-2009, und zuletzt Vladimir Kreck: Zur Diskussion gestellt: Autochthone Minderheiten und ökonomischer Mehrwert, in EJM 3-2011.

2 So ein Ergebnis der von der Europäischen Kommission 1996 in Auftrag gegebenen Studie „Euromosaic. Produktion und Reproduktion der Minderheiten-Sprachgemeinschaften in der Europäischen Union“. Näheres dazu bei Christoph Pan: Die Bedeutung von Minderheiten- und Sprachschutz für die kulturelle Vielfalt Europas, in EJM 1-2008.
} 
Zwar kann diese Kleinheit als solche nicht durch einen Willensakt der jeweiligen Gemeinschaften überwunden werden, um so mehr sind aber gerade solche Gruppen auf ihren sprachlich-kulturellen Zusammenhalt, ihre sprachlich-kulturelle Kohäsion angewiesen, die es ihnen ermöglicht, ihre Eigenheiten mittels sozialer Interaktion am Leben zu erhalten und zu pflegen und zugleich die daraus sich ergebenden spezifischen Interessen nach entsprechender interner Willensbildung auch nach außen wirksam zu vertreten.

Die Minderheit der Ladiner in Italien umfasst nur etwa 35.000 Angehörige und siedelt in einem administrativ dreigeteilten Gebiet mit jeweils unterschiedlich hohen Minderheitenschutz-Standards und kann schon aus diesen Gründen zu den in ihrer kulturellen Überlebensfähigkeit gefährdeten Minderheiten gezählt werden.

Ein Beispiel für eine beachtliche „Eigenleistung“ auch im Interesse der Stärkung der kulturellen Kohäsion der Ladiner bietet die im Jahr 2008 initiierte Volksabstimmung auf der Basis der 2001 geänderten italienischen Verfassung, durch welche drei derzeit noch der Region Veneto zugehörige ladinische Gemeinden mehrheitlich ihren Willen bekundet haben, administrativ zur autonomen Provinz Bozen (Region Trentino-Südtirol) zurückkehren zu wollen, der sie bis 1923 angehört hatten. ${ }^{3}$

Eine entsprechende Eigenleistung im Hinblick auf die Bildung einer gemeinsamen ladinischen Schriftsprache steht dagegen noch aus. So konnte das Dolomitenladinische bisher keine überlokale Schriftsprache herausbilden, vielmehr gibt es derzeit fünf verschiedene schriftliche Talschaftsidiome, von denen drei in neuerer Zeit besonders ausgebaut wurden. ${ }^{4}$ Zwar wurde darüber hinaus auch eine neue, gemeinsame Schriftsprache geschaffen, jedoch ist das Ladin Dolomitan bisher weder Amtsnoch Schulsprache im ladinischen Siedlungsgebiet und daher sprachwissenschaftlich als ,noch nicht durchgesetzte neue Schriftsprache“ zu qualifizieren.

Nicht zuletzt vor diesem Hintergrund erörtert Paul Videsott in seinem Beitrag die historischen Prozesse der Schriftsprachenbildung in Europa sowie die allgemeine Funktion der Schriftsprache, die

historisch deswegen entstanden ist, weil man gesprochene Sprache über Raum und Zeit konservieren wollte. Jeder, der das Bedürfnis hatte, dass seine Sprache möglichst Vielen [Hervorhebung d Verf] möglichst lange verständlich bleibt, war also bemüht, beim Schreiben jene Charakteristiken wegzulassen, von denen er ausgehen konnte, dass sie nur einem Teil seiner Leser zugänglich waren.

Da, wie Videsott weiter bemerkt, „die moderne Welt im Wesentlichen zu einer Welt der Schriftlichkeit geworden ist", erfüllt eine gemeinsame Schriftsprache somit auch die Funktion, die für kleine Minderheiten oft überlebenswichtige kulturelle Kohäsion in einem wesentlichen Bereich, jenem der schriftlichen Kommunikation, entscheidend zu stärken (und zugleich deren Installation zB als Amts- oder Schulsprache zu ermöglichen oder zu erleichtern) und die betreffende Sprache überhaupt erst zu einer „vollwertigen“ zu machen.

\footnotetext{
${ }^{3}$ Näheres dazu bei Davide Zaffi: Ladinische Volksabstimmung, in EJM 1-2008.

${ }^{4}$ Näheres dazu bei Paul Videsott: „Ladinische Einheit“ zwischen Anspruch und Wirklichkeit. Aktuelle Entwicklungen im Bereich der Sprachnormierung und des Sprachausbaus im Dolomitenladinischen, in EJM 4-2009.
} 
Dies wird auch dadurch untermauert, dass, wie Videsott darlegt, historische

Prozesse der modernen Nationenbildung und [solche] der Schriftsprachenbildung in Europa bisher meistens parallel verlaufen sind.

Die Tatsache, dass große wie kleinere (Kultur-)Nationen ihre Schriftsprachen bisher nach dem gleichen Muster und im Ergebnis mit mehr Vor- als Nachteilen herausgebildet haben, könnte den kleineren Sprachminderheiten in Europa wie zB den Ladinern womöglich ein Ansporn sein, diese Leistung im Interesse ihrer sprachlichkulturellen Fortexistenz ebenfalls zu erbringen und ihrer - teils schon existierenden - gemeinsamen Schriftsprache zum Durchbruch zu verhelfen.

Um eine kulturelle Kohäsion ganz anderer Art geht beim Haus des Deutschen Ostens (HDO) in München, das 1970 geschaffen wurde, um den nach dem Zweiten Weltkrieg aus verschiedenen Staaten Mittel- und Osteuropas geflüchteten oder vertriebenen Deutschen ein Zentrum für ,ostdeutsche Kulturarbeit“ zu bieten. Es geht beim HDO also ua darum, die infolge des politisch, geographisch, historisch und sprachlich-kulturell ursprünglich anderen Umfeldes spezifisch geprägte und ins kulturell-sprachliche „Mutterland“ in dieser Form „mitgebrachte“ deutsche Kultur nach Möglichkeit zu bewahren. In diesem Sinne und mit dieser Nuance setzt HDO-Direktor Ortfried Kotzian, der durch seinen Beitrag in das Projekt HDO, seine Ziele und Aktivitäten im einzelnen einführt, die EJM-Reihe „Vorstellung von Organisationen von und/oder für Minderheiten" fort.

Beate Sibylle Pfeil 